\title{
A collaborative and evolving response to the needs of frontline workers, patients and families during the COVID-19 pandemic at Tygerberg Hospital, Western Cape Province, South Africa
}

E Bröcker, ${ }^{1}$ MA Clinical Psychology (MPsych); K A Louw, ${ }^{1}$ MB ChB, FC Psych (SA), MMed (Psych), MPhil (Liaison Mental Health); M Hewett, ${ }^{1}$ MA Clinical Psychology (MPsych); H Burger, ${ }^{2}$ MB ChB, PG Dip Palliative Medicine, FC Rad Onc (SA); R Felix, ${ }^{3}$ MCur Hons (Mental Health Nursing), PG Dip Health Science Management, PG Dip Nursing Education, Registered Nurse (General, Psychiatric \& Community) and Midwife; P De Koker, ${ }^{4}$ MA Clinical Psychology (MPsych), MA Conflict \& Development; J Rossouw, ${ }^{1}$ MSc (Clinical Psychology), PhD (Psychiatry); S Seedat, ${ }^{1}$ MB ChB, FC Psych (SA), MMed (Psych), PhD

\author{
${ }^{1}$ Department of Psychiatry, Faculty of Medicine and Health Sciences, Stellenbosch University, Cape Town, South Africa \\ ${ }^{2}$ Division of Radiation Oncology, Department of Medical Imaging and Clinical Oncology, Tygerberg Academic Hospital and Faculty of Medicine \\ and Health Sciences, Stellenbosch University, Cape Town, South Africa. \\ ${ }^{3}$ Department of Nursing and Midwifery, Faculty of Medicine and Health Sciences, Stellenbosch University, Cape Town, South Africa \\ ${ }^{4}$ Desmond Tutu TB Centre, Department of Paediatrics and Child Health, Faculty of Medicine and Health Sciences, Stellenbosch University, \\ Cape Town, South Africa.
}

Corresponding author: E Bröcker (erineb@sun.ac.za)

\begin{abstract}
The global devastation caused by the COVID-19 pandemic and its mental health impact is undeniable. The physical and psychological consequences are wide-ranging - affecting patients fighting the disease, frontline workers in the trenches with them, healthcare staff deployed in high-care settings, and families disconnected from their loved ones in their darkest hours. Within 6 weeks of the COVID19 outbreak in South Africa, the Department of Psychiatry at Stellenbosch University established the TBH/SU COVID Resiliency Clinic to provide psychological support to frontline workers at Tygerberg Hospital. Identified barriers in healthcare workers accessing mental healthcare resulted in moving towards an on-site visibility to try to remove some of these barriers. This greater on-site presence enabled networking and building of relationships with frontline staff that over time highlighted other frontline needs, such as providing psychosocial and spiritual support to patients and their families. We share challenges, lessons learned and recommendations from two initiatives: the TBH/SU COVID-19 Resiliency Clinic, and an embedded COVID Care Team (CCT). We describe the establishment, roll-out and progress of the Clinic and the subsequent CCT.
\end{abstract}

S Afr Med J. Published online 23 March 2021. https://doi.org/10.7196/SAMJ.2021.v111i5.15648

The global devastation caused by the COVID-19 pandemic and its mental health impact is undeniable. The consequences are wideranging - affecting patients fighting the disease, frontline workers in the trenches with them, healthcare staff deployed in high-care settings, and families disconnected from their loved ones in their darkest hours.

COVID-19 has necessitated a rapid, co-ordinated and collaborative response from the healthcare system at large. ${ }^{[1]}$ Similarly, the response to the serious psychological sequelae precipitated by the pandemic has required a multipronged and multidisciplinary approach.

In this article we describe a collaborative mental health response to the COVID-19 pandemic led by the Department of Psychiatry in collaboration with the Division of Oncology at Tygerberg Hospital (TBH)/Stellenbosch University (SU), Cape Town, South Africa (SA), and volunteer providers. This response is characterised by two initiatives: the TBH/SU COVID-19 Resiliency Clinic and an embedded COVID Care Team (CCT). We describe the establishment, roll-out and progress of the Clinic and the subsequent CCT. Both of these initiatives were intended to augment existing staff and patient support services at $\mathrm{TBH}$.
We share our challenges and lessons learned in the hope that sharing these experiences will benefit other services and systems, especially in view of the threat of a looming third COVID-19 wave, acknowledging that our experiences may not be generalisable to all SA healthcare settings.

\section{The TBH/SU COVID-19 Resiliency Clinic}

Within 6 weeks of the COVID-19 outbreak in SA, the Department of Psychiatry at SU established the TBH/SU COVID Resiliency Clinic (hereafter referred to as Clinic) under the leadership of Prof. Soraya Seedat and Dr Jaco Rossouw to provide psychological support to frontline workers at TBH. This service is supported by 11 volunteer providers (psychiatrists and psychologists) who offer individual and group-based support. Initially, this service was solely virtual and mostly targeted TBH/SU frontline staff (e.g. COVID-19 intensive care unit (ICU)/high care), with clients accessing the service by voluntarily contacting the Clinic co-ordinator. The co-ordinator then connects clients to appropriate Clinic providers, who offer up to four sessions to each client. The service is centred on flexibility and 
confidentiality. Together, client and provider schedule sessions at a mutually convenient time to accommodate the challenging schedules of most frontline and essential healthcare workers, using a variety of platforms (phone call/WhatsApp call/MS Teams/Zoom). To ensure confidentiality, no Clinic notes are kept, and no feedback is given to employers. Since the providers are psychiatrists and psychologists, they determine further management of a client (e.g. referral to a clinical psychologist for psychotherapy), as needed. The flexible foursession approach of the Clinic is strongly rooted in Psychological First Aid (PFA) as described by the World Health Organization. ${ }^{[2]}$

Owing to the initial poor uptake by frontline staff, who were the target group, virtual support services were promoted to all TBH/ $\mathrm{SU}$ employees. It soon became apparent that, in order to overcome barriers to accessing care by frontline staff, the service needed to have greater on-site visibility. Barriers to help-seeking among healthcare workers have been widely documented and include fear of stigmatisation, a culture of silence, ingrained beliefs that self-care is selfish, work and time pressures, and a 'trenches mentality' (a culture of needing to be heroic and reluctance to approach providers who do not understand working in the trenches) ${ }^{[3-6]}$

Following consultation with the relevant stakeholders, hospital approval was obtained to set up an office close to the COVID-19 ICU/high-care area of TBH. This on-site visibility had a positive effect as more frontline staff, in particular, reached out to the Clinic (i.e. by coming to the office).

At the time of writing, the Clinic has provided on-site individual support sessions to many COVID-19 ICU/high-care staff and has also provided support groups, of which the majority were on site, largely for frontline staff.

The increased on-site presence of Clinic providers enabled networking and building of relationships with frontline staff (doctors, nurses, physiotherapists, dieticians, technicians, administrative support staff). Through these relationships other frontline needs, such as providing psychosocial and spiritual support to patients and their families, were identified to alleviate the burden on staff, facilitate prompt support and implement resilience-bolstering strategies.

\section{Identification of needs}

In addition to providing healthcare, frontline workers overnight became palliative care and family liaison and support providers. COVID-19-related infection control measures, resource constraints and anxiety related to exposure to infection limited the on-site services of routine auxiliary staff (such as social workers and pastors), adding to their burden. This situation was compounded by the no-visits policy at the hospital, isolation of patients from their families, and the psychological distress of family members, which added to their own distress. Frontline workers highlighted the need for more proactive psychosocial and spiritual patient support and family liaison support. An innovative approach to providing more holistic support to frontline workers, patients and families was therefore desperately needed. In an attempt to address this, a CCT (embedded in the Clinic) was formed to strengthen collaborative efforts. This team comprises a psychiatrist, two clinical psychologists, an oncologist, an advanced psychiatric nursing lecturer, a paediatrician, a research psychologist, psychiatry registrars and intern psychologists. A brief description of the daily co-ordinated activities of the CCT follows.

\section{Patient psychological support}

This entails proactive case finding of patients in need of psychological, psychiatric, spiritual or palliative care support. At the peak of the first wave of the pandemic, a psychiatrist visited the ICU daily to provide support to patients and connect them, via audio and video calls on a tablet, to family members and where indicated to spiritual or pastoral care. The demand increased beyond the capacity of a single psychiatrist during the second wave, and additional assistance was sought from a medical officer and an intern in the consultationliaison psychiatry team. Also, training of clinical psychologists based at TBH in the use of virtual/electronic platforms has helped to increase access to existing psychology services, overcome barriers relating to PPE use, and alleviate anxiety related to exposure to infection.

\section{Palliative care}

The need for the CCT to better understand the care needs of patients and families facing life-threatening illness and death was identified early in the response process. Currently, TBH does not have an established palliative care service, but doctors working in the Division of Radiation Oncology have the necessary training in and experience of providing clinical and psychosocial care to dying patients. Effective and empathic communication is one of the cornerstones of quality palliative care delivery, but COVID-19 significantly challenged normal modes of communication and care delivery. The CCT providers received training in dealing with endof-life communication scenarios from a senior palliative care-trained oncologist (author HB). This training enabled sensitive negotiation and management of these situations when they occurred (i.e. during a psychiatric consult or audio/video call) by the on-site team. During the second wave, increased patient numbers led to a growing need for palliative care support on general (non-intensive care) COVID-19 wards. Many patients with comorbidities, including advanced cancer, who developed moderate to severe COVID-19 symptoms were not eligible for intensive care. These patients required a holistic palliative care approach to support them through recovery and discharge or to ensure a dignified death. Doctors from the Division of Radiation Oncology became actively involved beyond the TBH ICU/high-care units in promoting and establishing an expanded model of palliative and supportive care. This model has oncologists on the ground supporting frontline workers in the provision of palliative symptom control and facilitating referral of patients to psychosocial services. The palliative care support model was greatly strengthened by the COVID-19 care guidelines and training videos published by the Association of Palliative Care Practitioners of South Africa (www. PALPRAC.org) and the Western Cape Department of Health (https:// coronavirus.westerncape.gov.za/health-workers).

\section{Connecting patients and their families}

Communication strategies had to be adapted to circumvent the restrictions on physical presence as well as the limitations on human resources during the height of the pandemic. During the first COVID-19 wave, a Double Robotics telepresence robot affectionately called 'Quintin' was made available to the CCT by the Stellenbosch University SUNskills laboratory, which enabled connection between patients and their families for audio and video calls. This service is currently offered 2 - 3 days a week by a clinical psychologist, intern clinical psychologist and psychiatric medical officer, who provide emotional support to families before and after these calls. This also allows families to say goodbye to loved ones and engage in spiritual rituals at the end of life. The current service allows for up to $10-15$ connections a week between patients and their families. Quintin operators developed a standard operating procedure that includes an outline of the role the operator plays in preparing the family before a conversation (i.e. what to expect and whether the call will be audio 
only or include visual). Frontline staff have a ward tablet available to connect patients and their families at times when a Quintin operator is not available. Quintin eased the workload of frontline staff, reduced the need for PPE, and freed up staff to perform other duties. The team has been continuously evaluating, adapting and researching ways to enhance and expand responsible and ethical use of Quintin, including data security measures.

\section{Extended family support}

The CCT assists frontline workers with referrals to a designated TBH social worker for bereavement counselling. As with other services, social workers experienced increasing work pressure owing to more patients dying compared with pre-COVID-19 times. To address this, an advanced psychiatry nursing lecturer on our team assists in providing telephonic counselling and support to patients and families remotely through the use of a cellphone or tablet.

\section{Assisting frontline staff with daily family updates}

Frontline workers routinely contact families to provide telephonic updates. The CCT has assisted with these calls where possible. A strict protocol is followed whereby the doctor concerned provides updates to the team member and advises on the information to be shared. When families request more information, this is communicated to the doctor concerned for further management. HospiVision (http://hospivision.org.za/) and pastoral care volunteers are currently being trained to assist with communicating with family members in anticipation of the burden of the third wave.

\section{Self-care in healthcare}

A digital mental health awareness messaging campaign was initiated. Two clinical psychologists from the Clinic worked with the TBH principal communications officer and developed material for a 'Self-Care in Healthcare' campaign to: (i) raise awareness of the importance of staff mental health; (ii) destigmatise mental health issues; (iii) promote help-seeking behaviour; and (iv) provide information on resources and services. Messages were distributed weekly via e-mail on topics that included 'Compassion fatigue and burnout', 'Stress and anxiety', 'Financial stress and job loss', 'Insomnia' and 'How do you know when you need help?', to name a few.

\section{Reflections}

On-site visibility allows providers to become part of the hospital team and to transition to the role of peer supporter. Having mental health and palliative professionals as part of the clinical team increases overall awareness of patients' psychosocial, spiritual and palliative care needs, reduces stigma and self-stigma, allows for sharing and holding of vulnerabilities in the face of trauma, and reduces barriers to accessing support and mental health treatment. Investment in these on-site mental health and palliative care providers will be important in the longer term, beyond the current pandemic. Table 1 provides a summary of recommendations based on the lessons learned from our shared experiences of both the Clinic and the CCT. Other systems may benefit from these recommendations in view of a looming third COVID-19 wave.

Table 1. Recommendations

\begin{tabular}{|c|c|}
\hline TBH/SU COVID Resiliency Clinic & Description \\
\hline Visibility & $\begin{array}{l}\text { On-site presence of providers builds relationships and trust and allows for a greater } \\
\text { understanding of the lived experience and needs of frontline workers and patients. }\end{array}$ \\
\hline Flexibility & $\begin{array}{l}\text { Frontline staff are facing unique challenges in terms of their schedules and resulting availability, } \\
\text { as well as their capacity to receive support. We advise flexibility in scheduling sessions - } \\
\text { flexibility in the approach to sessions based on the client needs, and flexibility in the delivery of } \\
\text { support (virtual/on site). Some clients who reach out may benefit from weekly check-ins, others } \\
\text { from the full four sessions as outlined in the Clinic PFA protocol. }\end{array}$ \\
\hline Empathy and patience & Meeting the client where he/she is at remains imperative. \\
\hline Provider support & Regular team check-ins and support spaces are needed to bolster provider resilience. \\
\hline Networking/collaboration & $\begin{array}{l}\text { The clinic does not function in isolation. The network of providers (psychologists/psychiatrists) } \\
\text { who made their skills available at reduced rates or pro bono enabled us to timeously connect } \\
\text { clients after the provision of PFA, when indicated. Collaboration with multidisciplinary } \\
\text { volunteers allowed the service to collectively expand and evolve to meet changing needs } \\
\text { through the different phases of the pandemic. }\end{array}$ \\
\hline COVID Care Model & Description \\
\hline Augment the existing structure and service & $\begin{array}{l}\text { Importantly, these initiatives sought to augment and support and not replace existing staff and } \\
\text { patient support structures that were stretched during the pandemic. }\end{array}$ \\
\hline Continuing needs assessment & $\begin{array}{l}\text { Networking and consulting with frontline staff on a regular basis remain key to the success of } \\
\text { this initiative. As the pandemic progressed and needs shifted, so too did our response. }\end{array}$ \\
\hline Sustainability & $\begin{array}{l}\text { The impact of COVID-19 is pervasive and long lasting, and care models need to consider the } \\
\text { sustainability of interventions that are provided. }\end{array}$ \\
\hline Innovation & $\begin{array}{l}\text { Providers need to be open to advances in technology and other creative ways to provide support } \\
\text { to patients, staff and families. }\end{array}$ \\
\hline On site mental health support & $\begin{array}{l}\text { Some clients accessed virtual support; however, increased on-site visibility further enabled } \\
\text { access. This could be considered in the longer term for staff mental wellness planning. }\end{array}$ \\
\hline Palliative care integration & $\begin{array}{l}\text { Palliative care skills have been critical. Greater integration and mainstreaming of palliative care } \\
\text { services could be considered in the planning of a longer-term integrated hospital care model. }\end{array}$ \\
\hline
\end{tabular}




\section{Conclusions}

The initiatives we describe are at the heart of providing a holistic intervention in a hospital setting. They seek to positively address cumulative and ongoing stress, trauma, uncertainty and loss faced by healthcare workers, patients and their families during the pandemic. Illustrating the potential impact of these initiatives is the following feedback received from Clinic providers:

'I have been part of the Clinic team for the past couple of months. The virtual sessions were new to me and I was not sure how this would impact on the client-therapist relationship and rapport building. And to my surprise, the shift to a symbolic holding space of emotional reflection, containment and meaning making of what is "visible" was more effective than I thought it would be. I assisted with on-site individual and group sessions as well, which have also been a very meaningful and growing experience. The frontline workers have been fighting this pandemic so hard and holding the pressure and pain does not come without a cost. To have been able to play - even a small part - in supporting them, has been a tremendous privilege. I commend the co-ordinators of the Resiliency Clinic for their very efficient and prompt way in responding to needs, connecting providers, and making this such a safe, confidential and enriching experience for everyone.'

'Accessing emotional support during the pandemic has been difficult for many people because they have been so busy and at times overwhelmed by their clinical responsibilities. Accessing help can also be uncomfortable for healthcare providers who are taught that they must cope even in adverse circumstances and that they must always put other people first. Taking care of our mental health and getting support when we need it should become a part of our culture and our work community. These brief Resiliency Clinic interventions can be very powerful for helping people get perspective, to strengthen existing coping mechanisms and to learn new tools to manage stress and emotional distress.'

\section{Declaration. None.}

Acknowledgements. We would like to pay tribute to all frontline workers during the COVID-19 pandemic for their tenacity and commitment to saving lives and upholding the dignity of patients at the end of life. We thank the volunteers and providers for making these initiatives viable, the Western Cape Provincial Department of Health and Tygerberg Hospital management for supporting these interventions, and the Stellenbosch University SUNskills laboratory for making Quintin the robot available to us. Lastly, we thank those who have donated funds to cover data costs to enable our work with Quintin. Without you, many calls and connections with families would not have been possible.

Author contributions. EB: contributed to conceptualisation, drafting of article, critical revision of content and approval of the version to be published. KAL: contributed to conceptualisation, critical revision of content and approval of the version to be published. $\mathrm{MH}$ : critical revision of content and approval of the version to be published. HB: contributed to conceptualisation, critical revision of content and approval of the version to be published. RF: critical revision of content and approval of the version to be published. PdK: critical revision of content and approval of the version to be published. JR: critical revision of content and approval of the version to be published. SS: contributed to conceptualisation, critical revision of content and approval of the version to be published.

Funding. EB is supported by funding received from the National Research Foundation Thuthuka Funding Scheme, the South African Medical Research Council (SAMRC) through its Division of Research Capacity Development under the SAMRC Bongani Mayosi National Health Scholars Programme of the South African National Treasury, and the SAMRC through the Extramural Genomics of Brain Disorders Unit. SS is supported by the South African Research Chairs Initiative in Posttraumatic Stress Disorder funded by the Department of Science and Technology and the National Research Foundation, and the SAMRC through the Extramural Genomics of Brain Disorders Unit. The content hereof is the sole responsibility of the authors and does not necessarily represent the official views of the funders.

\section{Conflicts of interest. None.}

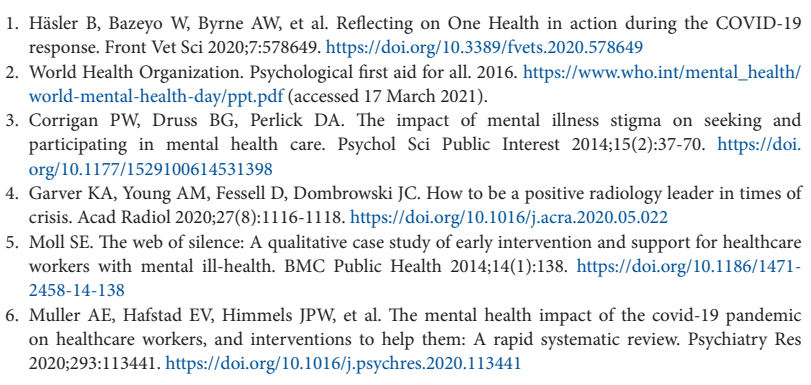

